\title{
ChemComm
}

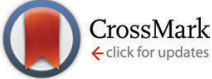

Cite this: Chem. Commun., 2017 53,1626

Received 2nd November 2016, Accepted 9th January 2017

DOI: $10.1039 / c 6 c c 08793 k$

www.rsc.org/chemcomm

\section{Hydrophobicity determines the fate of self-assembled fluorescent nanoparticles in cells $\dagger$}

\author{
Arthur H. A. M. van Onzen, ${ }^{a}$ Lorenzo Albertazzi, ${ }^{\text {ab }}$ Albertus P. H. J. Schenning, ${ }^{c}$ \\ Lech-Gustav Milroy ${ }^{a}$ and Luc Brunsveld*a
}

The fate of small molecule nanoparticles (SMNPs) composed of self-assembling intrinsically fluorescent $\pi$-conjugated oligomers was studied in cells as a function of side-chain hydrophobicity. While the hydrophobic SMNPs remained intact upon cellular uptake, the more hydrophilic SMNPs disassembled and dispersed throughout the cytosol.

Fluorescent nanoparticles hold great promise in cellular imaging, not least because of their excellent photophysical properties compared to single-dye imaging or single-dye functionalized nanoparticles. ${ }^{1,2}$ Small molecule nanoparticles (SMNPs) constructed from intrinsically fluorescent $\pi$-conjugated oligomers show additional promise ${ }^{3-6}$ because of their outstanding optical properties and their potential to circumvent the documented problems of solid or polymer-based nanoparticles such as high toxicity and low biodegradability. ${ }^{7}$ The preparation methods of self-assembled SMNPs enable efficient access to differently functionalized nanoparticles through the late-stage intermixing of diverse functionalized oligomers. ${ }^{4,8-12}$ More knowledge is needed of the interplay between these SMNPs and biological systems in order to understand, tune, and utilize their cellular stability and fate. ${ }^{13}$ The mode with which nanoparticles in general interact with and cross the cellular membrane greatly depends on nanoparticle size, surface charge, ${ }^{14}$ functional groups, ${ }^{15}$ hydrophobicity ${ }^{16-18}$ and geometry. ${ }^{19}$ Of special relevance for SMNPs is their integrity within the cytosol, where they encounter and interact with biomolecules that potentially affect

\footnotetext{
${ }^{a}$ Laboratory of Chemical Biology, Department of Biomedical Engineering and Institute of Complex Molecular Systems (ICMS), Eindhoven University of Technology, P.O. Box 513, 5600MB, Eindhoven, The Netherlands. E-mail: l.brunsveld@tue.nl

${ }^{b}$ Nanoscopy for Nanomedicine Group, Institute for Bioengineering of Catalonia (IBEC), Barcelona, 08028, Spain

${ }^{c}$ Laboratory of Functional Organic Materials and Devices, Department of Chemical Engineering and Chemistry and Institute of Complex Molecular Systems, Eindhoven University of Technology, P.O. Box 513, 5600MB, Eindhoven, The Netherlands

$\dagger$ Electronic supplementary information (ESI) available. See DOI: 10.1039/c6cc08793k
}

their self-assembled state. Control over the intracellular stability of SMNPs would help to optimize their imaging, targeting and clearance properties. While the fate of intrinsically fluorescent self-assembled SMNPs in cells has been less studied, their intrinsic fluorescence can in principle aid in monitoring molecular properties such as their stability - i.e. the balance between assembled and disassembled states. ${ }^{20}$ Here we show how tuning of side chain hydrophobicity controls intracellular integrity and the optical properties of fluorene oligomer based SMNPs in cells (Fig. 1).

We recently synthesized a library of fluorene-based oligomers with varying degrees of hydrophobicity by varying the side chains on the periphery of the chromophore (Fig. 1). ${ }^{21}$ The SMNPs prepared from these oligomers in water, ranging from 78 to $161 \mathrm{~nm}$ in size, exhibited varying dynamics. The content of ethylene glycol side chains correlated with nanoparticle dynamics: the more hydrophobic oligomers formed stable and compact SMNPs, while the more hydrophilic oligomers formed SMNPs that underwent exchange of the conjugated oligomers between the initially formed particles. ${ }^{21}$ We reasoned, therefore, that the high intrinsic fluorescence intensity of both the self-assembled nanoparticles and the free oligomers - owing to their extended $\pi$-conjugated core - combined with their aforementioned dynamic behaviour, would make them ideal test substrates to

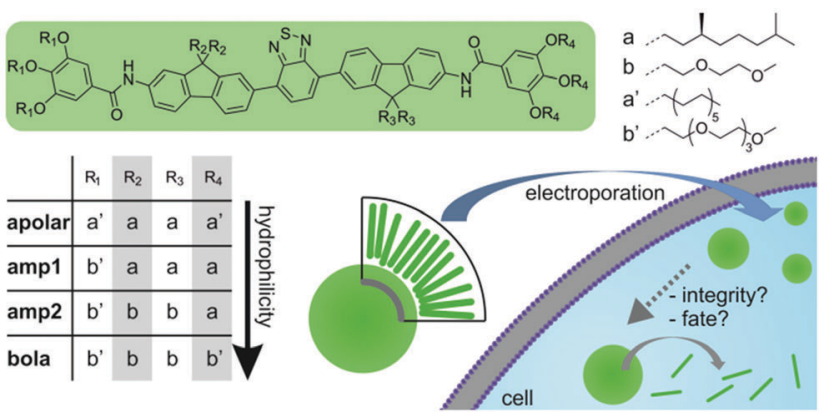

Fig. 1 Names and chemical structures of fluorene-based oligomers and SMNPs and their resulting cellular integrity. ${ }^{21}$ 
investigate the differentiated behaviour of these SMNPs in cells. The chromophore of the oligomer was found to be sensitive to polarity changes, as observed by emission-wavelength differences, and could therefore act as polarity probe. The characteristics of SMNPs composed of either apolar, amp1, amp2, and bola oligomers (Fig. 1) regarding their optical properties, including two-photon imaging, cellular stability, localization and responsive optical properties were therefore studied and related to their molecular characteristics.

Two-photon excitation microscopy measurements on SMNPs would provide an entry to monitor SMNPs in cells with little cellular background emission and photochemical damage. ${ }^{22}$ The applicability of this technique for the fluorene oligomer-based SMNPs was therefore first studied by recording the two-photon excitation and emission spectra of all SMNPs as aqueous-based samples (Fig. 2). While the two-photon excitation spectra $\left(\lambda_{\mathrm{em}}=480-580 \mathrm{~nm}\right)$ of all four types of SMNPs in water were similar in profile, the corresponding two-photon emission spectra $\left(\lambda_{2 \text {-photonex }}=725 \mathrm{~nm}\right)$ featured distinct differences. The maximum emission wavelength of apolar- and amp1-SMNPs was observed at $545 \mathrm{~nm}$, while for both amp2- and bola-SMNPs a red-shift in the maximum emission wavelength was measured to $580 \mathrm{~nm}$. The emission spectrum of bola-SMNPs is in this respect was even slightly more red-shifted compared to amp2-SMNPs. Collectively, the chromophores of these oligomers respond to a change in the polarity of the environment, with a red-shifted emission indicating a more hydrophilic environment. ${ }^{23}$ The SMNPs can thus be measured via two-photon excitation microscopy and the technique can be used to probe the polarity of the environment surrounding the oligomers via their emission spectrum.

We observed that our intrinsically fluorescent SMNPs were not aspecifically taken up by the commonly used immortalized HeLa cell line, in contrast to for example our studies on SMNPs functionalized with positively charged groups. ${ }^{12}$ We therefore elected to use electroporation to induce SMNP uptake in the cytosol and thus enable us to study the cellular integrity of the SMNPs in live cells. The apolar-SMNPs remained intact as nanoparticles inside the live cells, as judged by the presence of single, bright dots (SI 1, ESI $\dagger$ ). The HeLa cells typically contained a few apolar-SMNPs all located inside the cytosol, with no uptake into the nucleus. Indeed, the typical size of the apolar-SMNPs of circa $80 \mathrm{~nm}$ would preclude their passage into
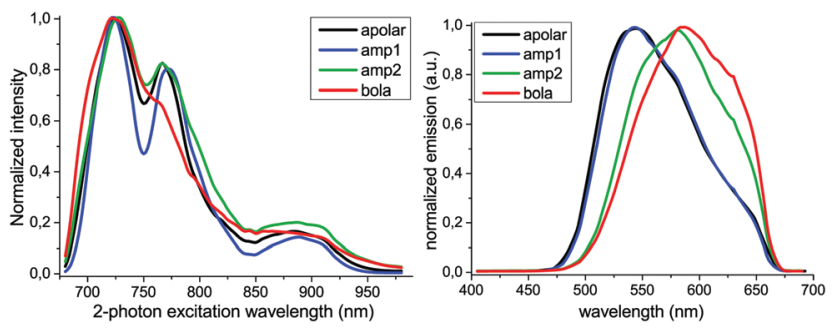

Fig. 2 Normalized two-photon excitation (left) and emission spectra $\left(\lambda_{\mathrm{ex}}=725 \mathrm{~nm}\right)$ (right) spectra of aqueous-based SMNP samples (fluorene oligomers listed in the insert) at $3 \mu \mathrm{M}$.

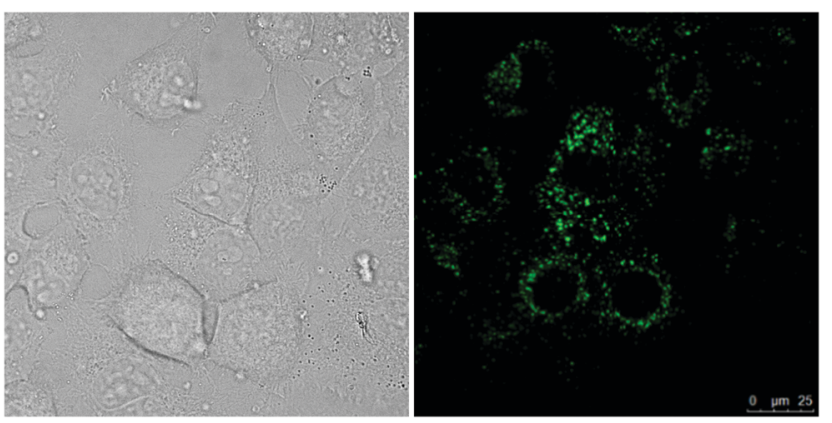

Fig. 3 Amp1-SMNPs electroporated into live HeLa cells: bright-field (left) and two-photon excitation fluorescence emission channel (right).

the nucleus as intact nanoparticles. ${ }^{24}$ In similar studies on the amp1-SMNPs the nanoparticles were clearly visible, perceptible as bright dots distributed throughout the cell, and particularly present around the nucleus (Fig. 3). Similar to the apolar-SMNPs, the nucleus remained free from amp1-SMNPs. The HeLa cells remained viable after SMNP electroporation judging by their characteristic morphology as seen by bright-field microscopy. The localization profile and particle characteristics of the amp1-SMNPs was similar to apolar-SMNPs, but the amount of fluorescent particles originating from the amp1-SMNP in cells was higher compared to the apolar-SMNPs. Time-lapse studies revealed that the location of the amp1-SMNPs within the cytosol slowly changed on the seconds time-scale (SI 2, ESI $\dagger$ ). Co-localization studies using Lyso-tracker ${ }^{\circledR}$ suggested that the amp1-SMNPs are not localized inside lysosomes but are instead freely available within the cytosol (SI 3, ESI $\dagger$ ), in line with the electroporation mode of delivery. ${ }^{25,26}$ This finding is potentially significant, as it raises the possibility of targeting cytosolic processes and biomolecules using specifically the amp1-SMNPs.

In subsequent two-photon microscopy studies on amp2-SMNPS (SI 4, ESI $\dagger$ ), the bright-field picture revealed characteristic HeLa morphology, but the confocal image at normal intensity setting did not reveal any detectible fluorescence. Upon increase of the laser intensity amp2-SMNPs became visible, but with concomitant cellular auto-fluorescence (SI 4, ESI $\dagger$ ). Compared to control, the amp2-SMNPs measured a 1.6-fold higher overall fluorescence. The lower fluorescence intensity of amp2-SMNPs compared to the amp1-SMNPs indicates an important influence of the side-chain hydrophobicity of the constituent oligomers on electroporation-dependent cellular particle uptake.

Bola-SMNPs were also electroporated with HeLa cells and subsequently imaged (Fig. 4, SI 5, ESI $\dagger$ ). Gratifyingly, the live HeLa cells exhibited a typical spreading and high fluorescence intensity. The area and number of cells successfully electroporated around the electroporation pin was 3-4 fold higher compared to the apolar- and amp1-SMNP experiments. Interestingly, and in contrast to the other SMNPs, fluorescence originating from the bola-SMNPs was detected abundantly and dispersed throughout the whole cell, including within the nucleus. In contrast to apolar- and amp1-SMNPs, no bright spots were observed in the cells. The bola-SMNPs are thus most probably disassembled 


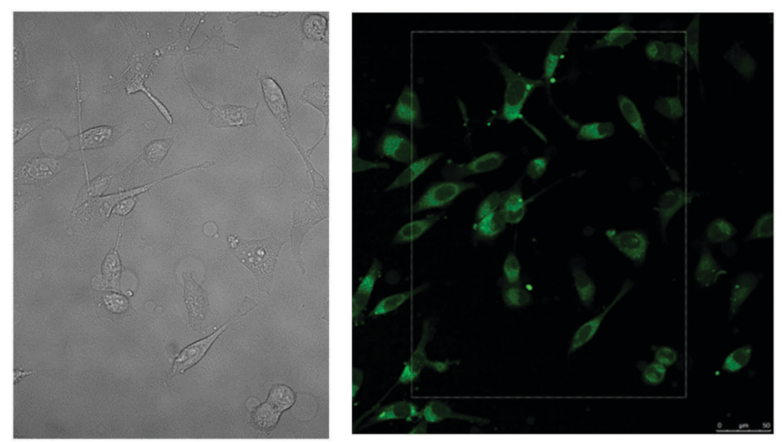

Fig. 4 Bola-SMNPs electroporated into live HeLa cells: bright-field (left), two-photon excitation fluorescence emission channel (right).

inside the cell. Co-localization studies on fixed cells with cetuximab-alexa647, which binds to the epidermal growth factor receptor (EGFR) present at membranes of HeLa-cells (SI 6, ESI $\dagger$ ), provided further proof that the bola-oligomers are localized within the cellular membrane boundary. ${ }^{27-29}$

The two-photon emission spectra of the SMNPs located within the live cells were recorded and compared to emission spectra recorded from aqueous-based samples (Fig. 5). The apolar- and amp1-SMNPs featured a similar maximum emission wavelength under both conditions. These results show that the hydrophobic environment in which the $\pi$-conjugated building blocks of these two types of intrinsically fluorescent selfassembled SMNPs find themselves inside the cells is the same as in bulk water, i.e. within a stable nanoparticle. ${ }^{21,30}$ This result is concordant with the microscopy observations, which showed that the particle integrity of apolar- and amp1-SMNPs remained intact inside cells. The bola-SMNPs however, displayed a significant shift in the maximum emission wavelength, from $585 \mathrm{~nm}$ in water to $545 \mathrm{~nm}$ inside the cells. In line with the observations made during the cellular imaging studies, the bola-SMNPs undergo a major change in integrity upon electroporation-dependent cellular
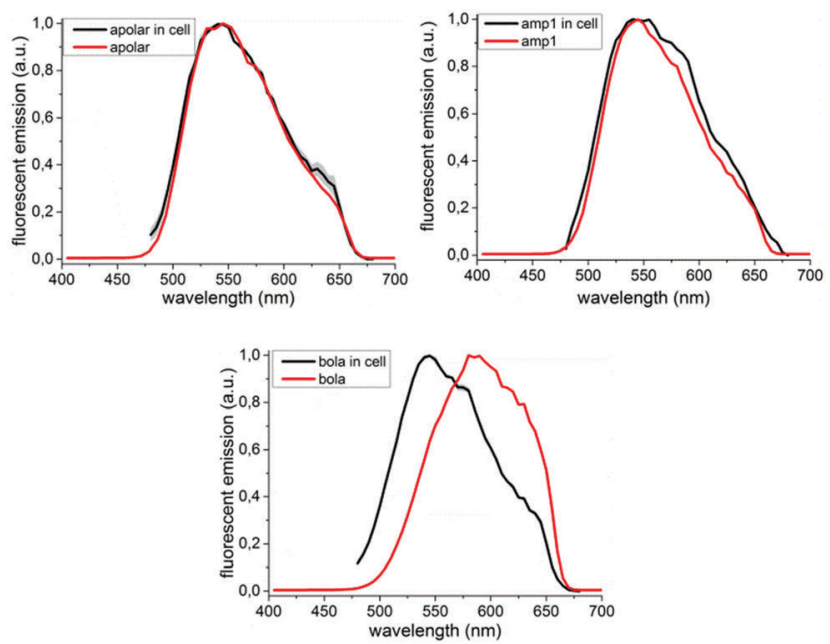

Fig. 5 Two-photon emission spectra of SMNPs recorded in live cells after electroporation (black) and in aqueous-based samples (red) of apolarSMNPs, amp1-SMNPs, and bola-SMNPs. uptake. The blue shift of the emission wavelength upon cellular uptake shows that the oligomers experience a more hydrophobic environment. ${ }^{23}$ The dynamic nature of the bola-SMNPs in combination with the biomolecular composition of the cell, thus appear to enable the bola-oligomers to disassemble and interact with hydrophobic components of the cell.

The subcellular distribution and accumulation of small molecules in cellular organelles is typically determined by their physical-chemical parameters. ${ }^{31,32}$ Studies have correlated these parameters to subcellular localization resulting in quantitative structure-activity decision rule models (QSAR). ${ }^{31,33-35}$ According to a decision-logic table ${ }^{35}$ the bola-oligomer should localize in the cytosol (potentially via protein binding), Golgi membranes and lipid droplets, because of the calculated $\log P$ value of $5.2^{36}$ and the overall neutral charge of the bola-oligomer. The higher hydrophobicity in these cellular domains, compared to bulk water, and hydrophobic driven interaction with biomolecules would explain the emission blue-shift of the chromophore of the bola-oligomer. ${ }^{31}$ The large size $\left(M_{\mathrm{w}}=2349 \mathrm{Da}\right)$ and the bolaamphiphilic character, however, mean that the bola-oligomer does not optimally follow the model criteria and that the predicted subcellular localization needs to be interpreted with caution. Nevertheless, the microscopy images of the HeLa cells with bola-SMNPs are in line with the localization of the bola-oligomer in these cellular compartments.

In conclusion, we report on a class of SMNPs composed of short synthetically tuneable $\pi$-conjugated oligomers, for which cellular uptake can easily be monitored by two-photon excitation microscopy. The hydrophobicity characteristics of the oligomers significantly impact the electroporation-dependent cellular fate of the SMNPs. The more hydrophobic apolar- and amp1-SMNPs remain intact as bright nanoparticles, localized in the cytosol. The most hydrophilic bola-SMNPs disassemble and are taken up into different cellular compartments, resulting in a segmented fluorescence throughout the cell. The intrinsically fluorescent core of the oligomers is sensitive to hydrophobicity changes, resulting in a blue shift in the fluorescence of the bola-SMNPs inside cells. The modular nature of these SMNPs, which allows both for the tuning of nanoparticle properties such as stability and optical features as well as for the easy incorporation of differently functionalized oligomers, enables fine-tuning of the resulting materials properties, thus opening up diverse opportunities for the imaging of biomolecular components of cells.

This work is supported by NanoNextNL, a micro and nanotechnology consortium of the government of The Netherlands and 130 partners and Netherlands Organisation for Scientific Research (NWO) via Gravity Program 024001035. LA acknowledges the AXA research fund.

\section{Notes and references}

1 O. S. Wolfbeis, Chem. Soc. Rev., 2015, 44, 4743-4768.

2 U. Resch-Genger, M. Grabolle, S. Cavaliere-Jaricot, R. Nitschke and T. Nann, Nat. Methods, 2008, 5, 763-775.

3 K. Li, W. Qin, D. Ding, N. Tomczak, J. Geng, R. Liu, J. Liu, X. Zhang, H. Liu, B. Liu and B. Z. Tang, Sci. Rep., 2013, 3, 1150.

4 X. Xu, R. Liu and L. Li, Chem. Commun., 2015, 51, 16733-16749. 
5 J. Schill, A. P. H. J. Schenning and L. Brunsveld, Macromol. Rapid Commun., 2015, 36, 1306-1321.

6 A. Kaeser and A. P. H. J. Schenning, Adv. Mater., 2010, 22, 2985-2997.

7 Andrey S. Klymchenko, J. Nanosci. Lett., 2013, 3, 1-8.

8 K. Petkau, A. Kaeser, I. Fischer, L. Brunsveld and A. P. H. J. Schenning, J. Am. Chem. Soc., 2011, 133, 17063-17071.

9 R. Weissleder, K. Kelly, E. Y. Sun, T. Shtatland and L. Josephson, Nat. Biotechnol., 2005, 23, 1418-1423.

10 M. Mammen, S.-K. Choi and G. M. Whitesides, Angew. Chem., Int. Ed., 1998, 37, 2754-2794.

11 X. Wang, F. He, L. Li, H. Wang, R. Yan and L. Li, ACS Appl. Mater. Interfaces, 2013, 5, 5700-5708.

12 I. Fischer, K. Petkau-Milroy, Y. L. Dorland, A. P. H. J. Schenning and L. Brunsveld, Chem. - Eur. J., 2013, 19, 16646-16650.

13 A. E. Nel, L. Mädler, D. Velegol, T. Xia, E. M. V. Hoek, P. Somasundaran, F. Klaessig, V. Castranova and M. Thompson, Nat. Mater., 2009, 8, 543-557.

14 R. Bajoria, S. R. Sooranna and S. F. Contractor, Hum. Reprod., 1997, 12, 1343-1348.

15 S. Ritz, S. Schöttler, N. Kotman, G. Baier, A. Musyanovych, J. Kuharev, K. Landfester, H. Schild, O. Jahn, S. Tenzer and V. Mailänder, Biomacromolecules, 2015, 16, 1311-1321.

16 A. M. Bannunah, D. Vllasaliu, J. Lord and S. Stolnik, Mol. Pharmaceutics, 2014, 11, 4363-4373.

17 K. Kobayashi, J. Wei, R. Iida, K. Ijiro and K. Niikura, Polym. J., 2014, 46, 460-468.

18 A. Gupta, D. Mandal, Y. Ahmadibeni, K. Parang and G. Bothun, Eur. Biophys. J., 2011, 40, 727-736.

19 H. Herd, N. Daum, A. T. Jones, H. Huwer, H. Ghandehari and C.-M. Lehr, ACS Nano, 2013, 7, 1961-1973.

20 X. Fan, Q. He, S. Sun, H. Li, Y. Pei and Y. Xu, Chem. Commun., 2016, 52, 1178-1181.
21 A. Kaeser, I. Fischer, R. Abbel, P. Besenius, D. Dasgupta, M. A. J. Gillisen, G. Portale, A. L. Stevens, L. M. Herz and A. P. H. J. Schenning, ACS Nano, 2013, 7, 408-416.

22 M. Pawlicki, H. A. Collins, R. G. Denning and H. L. Anderson, Angew. Chem., Int. Ed., 2009, 48, 3244-3266.

23 A. L. Stevens, A. Kaeser, A. P. H. J. Schenning and L. M. Herz, ACS Nano, 2012, 6, 4777-4787.

24 C. W. Pouton, K. M. Wagstaff, D. M. Roth, G. W. Moseley and D. A. Jans, Adv. Drug Delivery Rev., 2007, 59, 698-717.

25 P. E. Boukany, Y. Wu, X. Zhao, K. J. Kwak, P. J. Glazer, K. Leong and L. J. Lee, Adv. Healthcare Mater., 2014, 3, 682-689.

26 S.-W. Hui, in Electroporation Protocols, ed. S. Li, Humana Press, 2008, pp. 91-107.

27 K. Shigeta, T. Hayashida, Y. Hoshino, K. Okabayashi, T. Endo, Y. Ishii, H. Hasegawa and Y. Kitagawa, PLoS One, 2013, 8, e66302.

28 F. Zhang, S. Wang, L. Yin, Y. Yang, Y. Guan, W. Wang, H. Xu and N. Tao, Anal. Chem., 2015, 87, 9960-9965.

29 L. Albertazzi, B. Storti, L. Marchetti and F. Beltram, J. Am. Chem. Soc., 2010, 132, 18158-18167.

30 K.-Y. Pu, L. Cai and B. Liu, Macromolecules, 2009, 42, 5933-5940.

31 R. W. Horobin, in Organelle-Specific Pharmaceutical Nanotechnology, ed. V. Weissig and G. G. M. D'Souza, John Wiley \& Sons, Inc., 2010, pp. 193-206.

32 J. C. Stockert, A. Blázquez-Castro, M. Cañete, R. W. Horobin and A. Villanueva, Acta Histochem., 2012, 114, 785-796.

33 N. Zheng, H. N. Tsai, X. Zhang, K. Shedden and G. R. Rosania, Mol. Pharmaceutics, 2011, 8, 1611-1618.

34 N. Zheng, H. N. Tsai, X. Zhang and G. R. Rosania, Mol. Pharmaceutics, 2011, 8, 1619-1628.

35 R. W. Horobin, J. C. Stockert and F. Rashid-Doubell, Histochem. Cell Biol., 2013, 139, 623-637.

36 Calculated via PerkinElmer ChemBio3D v.14. 